\title{
Liouville theorems for a singular parabolic differential inequality with a gradient term
}

Zhong Bo Fang ${ }^{*}$ and Lijun Xu

\section{${ }^{*}$ Correspondence:}

fangzb7777@hotmail.com

School of Mathematical Sciences,

Ocean University of China, Qingdao,

266100, P.R. China

\begin{abstract}
In this paper, we study proofs of some new Liouville theorems for a strongly $p$-coercive quasi-linear parabolic type differential inequality with a gradient term and singular variable coefficients. The proofs are based on the test function method developed by Mitidieri and Pohozaev.
\end{abstract}

\section{Introduction}

We consider a singular quasi-linear parabolic differential inequality with a gradient term

$$
u_{t}-L u \geq a(x) u^{q}-b(x)|\nabla u|^{s}, \quad x \in \Omega, t>0,
$$

and the initial condition is given by

$$
u(x, 0)=u_{0}, \quad x \in \Omega
$$

where $q, s>0, \Omega \subset \mathbf{R}^{N}(N \geq 1)$ is a bounded domain with sufficiently smooth boundary or $\Omega=\mathbf{R}^{N}$, the initial function $u_{0} \in L_{\mathrm{loc}}^{1}(\Omega)$ is nonnegative, and the coefficients $a(x)$ and $b(x)$ are positive and singular on the boundary $\partial \Omega$ or at the origin 0 .

Let $A: \Omega \times \mathbf{R} \times \mathbf{R}^{N} \rightarrow \mathbf{R}$ be a Carathéodory function and define an operator $L$ as

$$
L u=\operatorname{div}(A(x, u, \nabla u) \nabla u),
$$

for any $u \in W_{\text {loc }}^{1,1}(\Omega \times(0, \infty))$ such that $A(x, u, \nabla u) \in L_{\text {loc }}^{1}(\Omega \times(0, \infty))$. The operator $L$ is said to be strongly $p$-coercive (S- $p$-C), if there exist three constants $c_{1}, c_{2}>0$ and $p>1$ such that the inequality

$$
c_{1}|\eta|^{p-2} \leq A(x, u, \eta) \leq c_{2}|\eta|^{p-2}
$$

holds for all $(x, u, \eta) \in \Omega \times \mathbf{R} \times \mathbf{R}^{N}$. For example, the Laplace operator is S-2-C and the $p$-Laplace operator is S- $p$-C.

Inequality (1.1) appears in many fields, such as fluid mechanics, biological species, and population dynamics, see [1-3]. From the perspective of fluid mechanics, (1.1) describes the non-Newtonian filtration phenomenon and the flow of gas in a porous medium, in which $a(x) u^{q}$ is called the source term and $-b(x)|\nabla u|^{s}$ is called the gradient absorption term.

O2014 Fang and Xu; licensee Springer. This is an Open Access article distributed under the terms of the Creative Commons Attribution License (http://creativecommons.org/licenses/by/2.0), which permits unrestricted use, distribution, and reproduction in any medium, provided the original work is properly cited. 
There have been many results on the nonexistence of nonnegative nontrivial global solutions for nonlinear parabolic type equations or inequalities (and systems), see [4-15] and references therein. In 1966, Fujita [4] studied the following Cauchy problem of the semilinear heat equation:

$$
\partial_{t} u-\Delta u=u^{p}, \quad x \in \mathbf{R}^{N}, t>0
$$

and he obtained the critical exponent $q_{c}=1+2 / N$ on the existence versus nonexistence of nonnegative nontrivial global solution. From then on, there have been a number of extensions of Fujita's results in many directions, and many scholars pay close attention to his results. The nonexistence of a global solution for nonlinear partial differential equations, which arises from the Liouville type theorem of harmonic function, is a nonlinear Liouville type theorem. Thus one can prove some properties of solutions in a bounded domain and this is a kind of essential reflection of blow-up or singularity theory as well, see [5] and references therein. In the whole space, Kartsatos [6] studied a Liouville type theorem on the solution for a quasi-linear parabolic equation without singular variable coefficient and gradient term. For a more general evolution and quasi-linear parabolic type inequality, see $[7,8]$ and references therein. By using the test function method, Mitidieri and Pohozaev $[9,10]$ studied the Liouville type theorems for elliptic and parabolic equations without gradient source term. Afterwards, by using the same method, Wei [11] obtained the nonexistence of the nonnegative nontrivial global weak solution for a semilinear parabolic inequality with a weight function $a(x, t) \geq\left(|x|^{2}+t\right)^{-\gamma}$, but without a gradient term. Note that the gradient term in the right-hand side of (1.1) will bring substantial difficulty to the research. In the whole space, Mitidieri and Pohozaev [12] put forward an important result for a differential inequality with a gradient nonlinearity; they considered the following quasi-linear elliptic inequality:

$$
-\Delta_{p} u \geq u^{q}|\nabla u|^{s}, \quad x \in \mathbf{R}^{N},
$$

and they proved that it has only constant solutions if $1<p<N, q>0, s \geq 0, q+s>p-1$, and $q+\frac{(N-1) s}{N-2}<\frac{N(p-1)}{N-p}$. Later, Karisti and Filippucci did further research on general forms of (1.4) (cf. [13, 14]). Galakhov [15] discussed a strongly $p$-coercive elliptic, parabolic, and hyperbolic type differential inequality with a gradient nonlinearity of constant coefficient in bounded and unbounded domains. Moreover, they obtained a Liouville theorem by making use of the test function method. Recently, $\mathrm{Li}$ and $\mathrm{Li}[16,17]$ extended their conclusions to the case of an elliptic type inequality with singular variable coefficients and obtained the nonexistence of solutions to that inequality and to the Harnack inequality. For singular parabolic problems in conical domains, refer to [18-20] and the references therein.

Besides the works mentioned above, there are few studies on nonlinear Liouville theorems for a parabolic type differential inequality (1.1) with singular variable coefficients and a gradient term. The purpose of this paper is to investigate the influence of an S$p$-C operator, the exponent of singular coefficients, and the gradient nonlinearity on the nonexistence of a nonnegative nontrivial global weak solution. The main difficulty lies in choosing a suitable test function according to different singularities of $a(x), b(x)$, and inequality (1.1) with gradient nonlinearity. We will use the test function technique, developed by Mitidieri and Pohozaev $[9,10,12]$, to show some Liouville theorems on the weak 
solution for problem (1.1)-(1.2) with bounded and unbounded domains. In particular, we recall that no use of comparison results and no conditions at infinity on the solution are required.

This paper is organized as follows: In Section 2, some preliminaries, such as some basic definitions and assumptions, and our main results are given. We prove the main results in Sections 3 and 4 .

\section{Preliminaries and main results}

Since $p>1$ and inequality (1.1) is degenerate or singular, problem (1.1)-(1.2) has no classical solution in general, and so it is reasonable to consider other solutions. We state the two definitions of a weak solution and of a solution with a positive lower bound.

Definition 1 A nonnegative function $u(x, t)$ is called a weak solution of problem (1.1)-(1.2) in $\mathbf{S}=\Omega \times(0, \infty)$, if the following two conditions are satisfied:

(i) $A(x, u, \nabla u) \nabla u \in L_{\mathrm{loc}}^{1}(\mathbf{S})$ and $u \in W_{\mathrm{loc}}^{1, p}(\mathbf{S}) \cap L_{\mathrm{loc}}^{q}(\mathbf{S})$,

(ii) for any nonnegative function $\varphi \in C_{0}^{1}(\mathbf{S})$, we have

$$
\begin{aligned}
\int_{\mathbf{S}} a(x) u^{q} \varphi d x d t \leq & -\int_{\Omega} u_{0} \varphi(x, 0) d x-\int_{\mathbf{S}} u \partial_{t} \varphi d x d t \\
& +\int_{\mathbf{S}} A(x, u, \nabla u) \nabla u \nabla \varphi d x d t+\int_{\mathbf{S}} b(x)|\nabla u|^{s} \varphi d x d t
\end{aligned}
$$

Definition 2 A nonnegative function $u \in W_{\mathrm{loc}}^{1, p}(\mathbf{S}) \cap L_{\mathrm{loc}}^{q}(\mathbf{S})$ such that $A(x, u, \nabla u) \nabla u \in$ $L_{\text {loc }}^{1}(\mathbf{S})$ is said to be a solution with positive lower bound in $\mathbf{S}$, if there exists $c>0$ such that $u(x, t) \geq c$ a.e. in $\mathbf{S}$.

We consider two types of singularities.

Type 1: Both $a(x)$ and $b(x)$ are singular on the boundary $\partial \Omega$, i.e., there exist constants $c_{0}, d_{0}>0$ and $\alpha, \beta \in \mathbf{R}$ such that

$$
a(x) \geq c_{0} \rho(x)^{-\alpha}, \quad b(x) \geq d_{0} \rho(x)^{-\beta}, \quad x \in \Omega^{\varepsilon},
$$

where $\rho(x)=\operatorname{dist}(x, \partial \Omega)$ and $\Omega^{\varepsilon}=\{x \in \Omega: \rho(x) \leq \varepsilon\}$ for sufficiently small $\varepsilon>0$.

Type 2: Both $a(x)$ and $b(x)$ are singular at the origin in the case that $\Omega=\mathbf{R}^{N}$, i.e., there exist constants $c_{1}, d_{1}>0$ and $\alpha, \beta \in \mathbf{R}$ such that

$$
a(x) \geq c_{1}|x|^{-\alpha}, \quad b(x) \geq d_{1}|x|^{-\beta}, \quad x \in \mathbf{R}^{N} \backslash\{0\} .
$$

To establish a priori estimates of the solutions, we define some test functions which have the form of a separation of the variables, and those functions will be widely used in the sequel.

For the space variable and singular Type 1 , we introduce a cut-off function $\xi_{\varepsilon} \in$ $C_{0}^{1}(\Omega ;[0,1])$ that satisfies

$$
0 \leq \xi_{\varepsilon}(x) \leq 1, \quad \xi_{\varepsilon}(x)=1 \quad \text { in } \Omega \backslash \Omega^{2 \varepsilon}, \quad \xi_{\varepsilon}(x)=0 \quad \text { in } \Omega^{\varepsilon},
$$


and

$$
\left|\nabla \xi_{\varepsilon}\right| \leq c \varepsilon^{-1}, \quad x \in \Omega,
$$

for some constant $c>0$.

For the space variable and singular Type 2, we consider a cut-off function $\xi_{R} \in$ $C_{0}^{1}\left(\mathbf{R}^{N} ;[0,1]\right)$ that satisfies

$$
0 \leq \xi_{R}(x) \leq 1, \quad \xi_{R}(x)=1 \quad \text { in } B_{R}(0), \quad \xi_{R}(x)=0 \quad \text { in } \mathbf{R}^{N} \backslash B_{2 R}(0),
$$

and

$$
\left|\nabla \xi_{R}\right| \leq \check{c} R^{-1}, \quad x \in \Omega
$$

for some constant $\check{c}>0$.

Set

$$
\chi(x)=\xi^{\lambda},
$$

where $\lambda>0$ is a parameter to be chosen later according to the nature of problem (1.1)-(1.2).

For the time variable, assume that $\eta(t) \in C^{\infty}([0, \infty))$ and

$$
0 \leq \eta(t) \leq 1, \quad \eta(t)=1 \quad \text { in }[0,1], \quad \eta(t)=0 \quad \text { in }[2, \infty], \quad-C \leq \eta^{\prime}(t) \leq 0 .
$$

Then, by appropriate combinations of the above functions, one can choose suitable test functions and obtain the following nonlinear Liouville theorems.

Theorem 1 Let $p>1, q>\max \left\{1, p-1, \frac{s}{p-s}\right\}$, and let $s \in\left(0, \frac{p q}{q+1}\right)$. Suppose that $A$ is $S-p-C$ and $a, b: \Omega \rightarrow \mathbf{R}_{+}$(or $a, b: \mathbf{R}^{N} \backslash\{0\} \rightarrow \mathbf{R}_{+}$) are continuous functions that satisfy (2.2) (or (2.3)).

(I) When both $a(x)$ and $b(x)$ are singular on $\partial \Omega$, if

$$
\max \{r, q-1\}<\theta<q, \quad \alpha>\frac{\theta p-r}{\theta-r}, \quad \beta<\frac{q(p-s)-s+\alpha[(p-s)(\theta-q)+s]}{\theta q},
$$

then any nonnegative weak solution to problem (1.1)-(1.2) is trivial, i.e., $u(x, t)=0$ a.e. in $\mathbf{S}$.

(II) When both $a(x)$ and $b(x)$ are singular at 0 , we consider the following two conditions:

(i)

$$
\begin{aligned}
& \max \left\{r, q-1, \frac{N r}{p}\right\}<\theta<q, \quad \alpha<\frac{N(1-q)}{\theta+1-q}, \\
& \beta>\frac{N[q(p-s)-s]+\alpha[(p-s)(\theta-q)+s]}{\theta q}
\end{aligned}
$$

(ii)

$$
\begin{aligned}
& \max \{r, q-1\}<\theta<\min \left\{q, \frac{N r}{p}\right\}, \quad \alpha<\min \left\{\frac{N(1-q)}{\theta+1-q}, \frac{\theta p-N r}{\theta-r}\right\}, \\
& \beta>\frac{N[q(p-s)-s]+\alpha[(p-s)(\theta-q)+s]}{\theta q} .
\end{aligned}
$$


If one of the conditions (i) and (ii) holds, then any nonnegative weak solution to problem (1.1)-(1.2) is trivial, i.e., $u(x, t)=0$ a.e. in $\mathbf{S}$, where $\theta$ is a parameter and $r=q+1-p$.

Theorem 2 Suppose that the assumptions of Theorem 1 are satisfied. Then problem (1.1)(1.2) has no solution with positive lower bound in $\mathbf{S}$, if

$$
\alpha>p \quad \text { or } \quad \beta<\alpha-\frac{\alpha s}{p}
$$

when both $a(x)$ and $b(x)$ are singular on $\partial \Omega$, or if

$$
\alpha<p \quad \text { or } \quad \beta>\alpha-\frac{\alpha s}{p}
$$

when both $a(x)$ and $b(x)$ are singular at 0 .

Remark 1 If inequality (1.1) is modified with

$$
u_{t}+L u \geq a(x) u^{q}-b(x)|\nabla u|^{s}, \quad x \in \Omega, t>0,
$$

one can obtain the following results by a similar argument in the proof of Theorem 1.

Suppose that the assumptions of Theorem 1 are satisfied.

(I') When both $a(x)$ and $b(x)$ are singular on $\partial \Omega$, if

$$
\theta>q, \quad \alpha>\frac{\theta p-r}{\theta-r}, \quad \beta<\frac{q(p-s)-s+\alpha[(p-s)(\theta-q)+s]}{\theta q},
$$

then any nonnegative weak solution to problem (1.1a)-(1.2) is trivial, i.e., $u(x, t)=0$ a.e. in $\mathbf{S}$.

(II') When both $a(x)$ and $b(x)$ are singular at 0 , we consider the following two conditions:

$\left(i^{\prime}\right)$

$$
\begin{aligned}
& \theta>\max \left\{q, \frac{N r}{p}\right\}, \quad \alpha<\frac{N(1-q)}{\theta+1-q}, \\
& \beta>\frac{N[q(p-s)-s]+\alpha[(p-s)(\theta-q)+s]}{\theta q},
\end{aligned}
$$

$\left(\mathrm{ii}^{\prime}\right)$

$$
\begin{aligned}
& q<\theta<\frac{N r}{p}, \quad \alpha<\min \left\{\frac{N(1-q)}{\theta+1-q}, \frac{\theta p-N r}{\theta-r}\right\}, \\
& \beta>\frac{N[q(p-s)-s]+\alpha[(p-s)(\theta-q)+s]}{\theta q} .
\end{aligned}
$$

If one of the conditions ( $\left.\mathrm{i}^{\prime}\right)$ and (ii') holds, then any nonnegative weak solution to problem (1.1a)-(1.2) is trivial, i.e., $u(x, t)=0$ a.e. in $\mathbf{S}$, where $\theta$ is a parameter and $r=q+1-p$.

Remark 2 Since Theorem 2 is independent of the parameter $\theta$, one can also obtain the same results for problem (1.1a)-(1.2). 
Fang and Xu Journal of Inequalities and Applications 2014, 2014:62

Page 6 of 12

http://www.journalofinequalitiesandapplications.com/content/2014/1/62

3 The proof of Theorem 1

Our proof mainly consists of three steps.

Step 1. For $T>0$, define $Q_{\Omega, T}:=\Omega \times[0,2 T]$ and let $\psi(x, t)=\eta_{T}(t) \chi(x)$ be a cutoff funcion, where $\chi(x)$ is defined as (2.6) and $\eta_{T}(t)=\eta(t / T)$. Clearly, we get

$$
-\frac{C}{T} \leq \frac{\partial \eta_{T}(t)}{\partial t} \leq 0
$$

For $k>1$, which will be determined later, and $d=q-\theta>0$, multiplying both sides of (1.1) with a test function $\Psi=\psi^{k} u^{-d}>0$ and integrating the result over $Q_{\Omega, T}$, we have

$$
\begin{aligned}
& \int_{Q_{\Omega, T}} a(x) u^{q-d} \psi^{k} d x d t \\
& \leq-\int_{\Omega} u_{0}^{1-d} \chi^{k}(x) d x-\int_{Q_{\Omega, T}} \frac{1}{1-d} u^{1-d} \frac{\partial \psi^{k}}{\partial t} d x d t \\
& \quad+\int_{Q_{\Omega, T}}(A(x, u, \nabla u) \nabla u) \nabla u^{-d} \psi^{k} d x d t+\int_{Q_{\Omega, T}}(A(x, u, \nabla u) \nabla u) u^{-d} \nabla \psi^{k} d x d t \\
& \quad+\int_{Q_{\Omega, T}} b(x)|\nabla u|^{s} u^{-d} \psi^{k} d x d t .
\end{aligned}
$$

It follows from (1.3) that

$$
\begin{aligned}
& \int_{Q_{\Omega, T}} a(x) u^{\theta} \psi^{k} d x d t+\int_{\Omega} u_{0}^{1-d}(x) \chi^{k}(x) d x \\
& \leq \int_{Q_{\Omega, T}} \frac{1}{d-1} u^{1-d} \frac{\partial \psi^{k}}{\partial t} d x d t-c_{1} d \int_{Q_{\Omega, T}} u^{-d-1}|\nabla u|^{p} \psi^{k} d x d t \\
& \quad+c_{2} k \int_{Q_{\Omega, T}} u^{-d}\left(|\nabla u|^{p-2} \nabla u\right) \psi^{k-1}|\nabla \psi| d x d t+\int_{Q_{\Omega, T}} b(x)|\nabla u|^{s} u^{-d} \psi^{k} d x d t .
\end{aligned}
$$

By the definition of $\psi$, we get

$$
\begin{aligned}
\int_{Q_{\Omega, T}} a(x) u^{\theta} \psi^{k} d x d t+\int_{\Omega} u_{0}^{1-d}(x) \chi^{k}(x) d x+c_{1} d \int_{Q_{\Omega, T}} u^{-d-1}|\nabla u|^{p} \psi^{k} d x d t \\
\leq \frac{C}{T} \int_{Q_{\Omega, T}} u^{1-d} \psi^{k-1} d x d t+c_{2} k \int_{Q_{\Omega, T}} u^{-d}|\nabla u|^{p-1} \psi^{k-1}|\nabla \psi| d x d t \\
\quad+\int_{Q_{\Omega, T}} b(x)|\nabla u|^{s} u^{-d} \psi^{k} d x d t .
\end{aligned}
$$

By applying Young's inequality to the three terms in the right-hand side of (3.1), we obtain

$$
\begin{aligned}
& \frac{C}{T} \int_{Q_{\Omega, T}} u^{1-d} \psi^{k-1} d x d t \\
& \quad \leq \frac{1}{4} \int_{Q_{\Omega, T}} a(x) u^{\theta} \psi^{k} d x d t+C T^{\frac{-\theta}{q-1}} \int_{Q_{\Omega, T}} a(x)^{\frac{q-\theta-1}{q-1}} \psi^{k-\frac{\theta}{q-1}} d x d t \\
& c_{2} k \int_{Q_{\Omega, T}} u^{-d}|\nabla u|^{p-1} \psi^{k-1}|\nabla \psi| d x d t \\
& \quad \leq \frac{c_{1} d}{4} \int_{Q_{\Omega, T}} u^{-d-1}|\nabla u|^{p} \psi^{k} d x d t+C \int_{Q_{\Omega, T}} u^{\theta-r} \psi^{k-p}|\nabla \psi|^{p} d x d t,
\end{aligned}
$$


Fang and Xu Journal of Inequalities and Applications 2014, 2014:62

Page 7 of 12

http://www.journalofinequalitiesandapplications.com/content/2014/1/62

where $r=q+1-p$,

$$
\begin{aligned}
& \int_{Q_{\Omega, T}} b(x)|\nabla u|^{s} u^{-d} \psi^{k} d x d t \\
& \quad \leq \frac{c_{1} d}{4} \int_{Q_{\Omega, T}} u^{-d-1}|\nabla u|^{p} \psi^{k} d x d t+C \int_{Q_{\Omega, T}} b(x)^{\frac{p}{p-s}} u^{\frac{(p-s)(\theta-q)+s}{p-s}} \psi^{k} d x d t .
\end{aligned}
$$

Since $d>0,0<1-d=1-q+\theta<\theta, 0<\theta-r<\theta$, and $\frac{(p-s)(\theta-q)+s}{p-s}<s$, we know that

$$
\max \{r, q-1\}<\theta<q, \quad q>\max \left\{1, p-1, \frac{s}{p-s}\right\}, \quad s \in\left(0, \frac{p q}{q+1}\right) .
$$

Applying Young's inequality to the two terms in the right-hand side of (3.3) and (3.4) yields

$$
\begin{aligned}
& C \int_{Q_{\Omega, T}} u^{\theta-r} \psi^{k-p}|\nabla \psi|^{p} d x d t \\
& \quad \leq \frac{1}{4} \int_{Q_{\Omega, T}} a(x) u^{q-d} \psi^{k} d x d t+C \int_{Q_{\Omega, T}} a(x)^{\frac{r-\theta}{r}} \psi^{\frac{k r-p \theta}{r}}|\nabla \psi|^{\frac{\theta p}{r}} d x d t \\
& C \int_{Q_{\Omega, T}} b(x)^{\frac{p}{p-s}} u^{\frac{(p-s)(\theta-q)+s}{p-s}} \psi^{k} d x d t \\
& \quad \leq \frac{1}{4} \int_{Q_{\Omega, T}} a(x) u^{\theta} \psi^{k} d x d t+C \int_{Q_{\Omega, T}} a(x)^{\frac{-(p-s)(\theta-q)-s}{q(p-s)-s}} b(x)^{\frac{\theta p}{q(p-s)-s}} \psi^{k} d x d t
\end{aligned}
$$

Let $k$ be large enough such that $k>\max \left\{\frac{\theta}{q-1}, p, \frac{p \theta}{r}\right\}$. From inequalities (3.1)-(3.6), we deduce

$$
\begin{aligned}
& \frac{1}{4} \int_{Q_{\Omega, T}} a(x) u^{\theta} \psi^{k} d x d t+\int_{\Omega} u_{0}^{1-d}(x) \chi^{k}(x) d x+\frac{c_{1} d}{2} \int_{Q_{\Omega, T}} u^{-d-1}|\nabla u|^{p} \psi^{k} d x d t \\
& \leq C T^{\frac{-\theta}{q-1}} \int_{Q_{\Omega, T}} a(x)^{\frac{q-\theta-1}{q-1}} \psi^{k-\frac{\theta}{q-1}} d x d t+C \int_{Q_{\Omega, T}} a(x)^{\frac{r-\theta}{r}} \psi^{\frac{k r-p \theta}{r}}|\nabla \psi|^{\frac{\theta p}{r}} d x d t \\
& \quad+C \int_{Q_{\Omega, T}} a(x)^{\frac{-(p-s)(\theta-q)-s}{q(p-s)-s}} b(x)^{\frac{\theta p}{q(p-s)-s}} \psi^{k} d x d t .
\end{aligned}
$$

We then have

$$
\begin{aligned}
& \int_{0}^{T} \int_{\Omega} a(x) u^{\theta} \chi k d x d t \\
& \leq C T^{\frac{-\theta}{q-1}} \int_{0}^{T} \int_{\Omega} a(x)^{\frac{q-\theta-1}{q-1}} \chi^{k-\frac{\theta}{q-1}} d x d t+C \int_{0}^{T} \int_{\Omega} a(x)^{\frac{r-\theta}{r}} \chi^{\frac{k r-p \theta}{r}}|\nabla \chi|^{\frac{\theta p}{r}} d x d t \\
& \quad+C \int_{0}^{T} \int_{\Omega} a(x)^{\frac{-(p-s)(\theta-q)-s}{q(p-s)-s}} b(x)^{\frac{\theta p}{q(p-s)-s}} \chi^{k} d x d t .
\end{aligned}
$$


Fang and Xu Journal of Inequalities and Applications 2014, 2014:62

Page 8 of 12

http://www.journalofinequalitiesandapplications.com/content/2014/1/62

It can be seen that $|\nabla \chi|^{\frac{\theta p}{r}}=\lambda^{\frac{\theta p}{r}} \xi^{\frac{\theta p(\lambda-1)}{r}}|\nabla \xi|^{\frac{\theta p}{r}}$, and we take $\lambda>\max \left\{1, \frac{\theta p}{k r}\right\}$. We then obtain

$$
\begin{aligned}
\int_{0}^{T} & \int_{\Omega} a(x) u^{\theta} \chi k d x d t \\
\leq & C T^{\frac{-\theta}{q-1}} \int_{0}^{T} \int_{\operatorname{supp}|\nabla \xi|} a(x)^{\frac{q-\theta-1}{q-1}} \xi^{\lambda\left(k-\frac{\theta}{q-1}\right)} d x d t \\
& +C \lambda^{\frac{\theta p}{r}} \int_{0}^{T} \int_{\operatorname{supp}|\nabla \xi|} a(x)^{\frac{r-\theta}{r}} \xi^{\frac{\lambda(k r-p \theta)}{r}}|\nabla \xi|^{\frac{\theta p}{r}} d x d t \\
& +C \int_{0}^{T} \int_{\operatorname{supp}|\nabla \xi|} a(x)^{\frac{-(p-s)(\theta-q)-s}{q(p-s)-s}} b(x)^{\frac{\theta p}{q(p-s)-s}} \xi^{\lambda k} d x d t \\
\leq & C T^{\frac{-\theta}{q-1}} \int_{0}^{T} \int_{\operatorname{supp}|\nabla \xi|} a(x)^{\frac{q-\theta-1}{q-1}} d x d t+C \lambda^{\frac{\theta p}{r}} \int_{0}^{T} \int_{\text {supp }|\nabla \xi|} a(x)^{\frac{r-\theta}{r}}|\nabla \xi|^{\frac{\theta p}{r}} d x d t \\
& +C \int_{0}^{T} \int_{\operatorname{supp}|\nabla \xi|} a(x)^{\frac{-(p-s)(\theta-q)-s}{q(p-s)-s}} b(x)^{\frac{\theta p}{q(p-s)-s}} d x d t .
\end{aligned}
$$

Step 2. When both $a(x)$ and $b(x)$ are singular on $\partial \Omega$, we take $\xi=\xi_{\varepsilon}$ in (3.7). Evidently, one has

$$
\operatorname{supp}|\nabla \xi|=\Omega^{2 \varepsilon} \backslash \Omega^{\varepsilon}=\{x \in \Omega: \varepsilon \leq \rho(x) \leq 2 \varepsilon\} .
$$

By (2.2), we get

$$
\begin{aligned}
& \int_{0}^{T} \int_{\Omega} a(x) u^{\theta} \xi^{k \lambda} d x d t \\
& \leq C T^{\frac{-\theta}{q-1}} \int_{0}^{T} \int_{\Omega^{2 \varepsilon} \backslash \Omega^{2 \varepsilon}} \rho(x)^{\frac{-\alpha(q-\theta-1)}{q-1}} d x d t+C \lambda^{\frac{\theta p}{r}} \int_{0}^{T} \int_{\Omega^{2 \varepsilon} \backslash \Omega^{2 \varepsilon}} \rho(x)^{\frac{-\alpha(r-\theta)}{r}}\left|\nabla \xi_{\varepsilon}\right|^{\frac{\theta p}{r}} d x d t \\
& \quad+C \int_{0}^{T} \int_{\Omega^{2 \varepsilon} \backslash \Omega^{2 \varepsilon}} \rho(x)^{\frac{\alpha[(p-s)(\theta-q)+s]-\beta \theta p}{q(p-s)-s}} d x d t
\end{aligned}
$$

By a standard change of variables and (2.4), we then obtain

$$
\begin{aligned}
\int_{0}^{T} & \int_{\Omega \backslash \Omega^{2 \varepsilon}} a(x) u^{\theta} d x d t \\
\leq & \int_{0}^{T} \int_{\Omega} a(x) u^{\theta} \xi^{k \lambda} d x d t \\
\leq & C T^{\frac{-\theta}{q-1}} \int_{0}^{T} \int_{\Omega^{2 \varepsilon} \backslash \Omega^{2 \varepsilon}} \rho(x)^{\frac{-\alpha(q-\theta-1)}{q-1}} d x d t+C \lambda^{\frac{\theta p}{r}} \int_{0}^{T} \int_{\Omega^{2 \varepsilon} \backslash \Omega^{2 \varepsilon}} \rho(x)^{\frac{-\alpha(r-\theta)}{r}}\left|\nabla \xi_{\varepsilon}\right|^{\frac{\theta p}{r}} d x d t \\
& +C \int_{0}^{T} \int_{\Omega^{2 \varepsilon} \backslash \Omega^{2 \varepsilon}} \rho(x)^{\frac{\alpha[(p-s)(\theta-q)+s]-\beta \theta p}{q(p-s)-s}} d x d t \\
= & C T^{\frac{-\theta}{q-1}} \int_{0}^{T} \int_{\{x \in \Omega: \varepsilon \leq \rho(x) \leq 2 \varepsilon\}} \rho(x)^{\frac{-\alpha(q-\theta-1)}{q-1}} d x d t \\
& +C \lambda^{\frac{\theta p}{r}} \int_{0}^{T} \int_{\{x \in \Omega: \varepsilon \leq \rho(x) \leq 2 \varepsilon\}} \rho(x)^{\frac{-\alpha(r-\theta)}{r}}\left|\nabla \xi_{\varepsilon}\right|^{\frac{\theta p}{r}} d x d t
\end{aligned}
$$




$$
\begin{aligned}
& +C \int_{0}^{T} \int_{\{x \in \Omega: \varepsilon \leq \rho(x) \leq 2 \varepsilon\}} \rho(x)^{\frac{\alpha[(p-s)(\theta-q)+s]-\beta \theta p}{q(p-s)-s}} d x d t \\
\leq & C T^{\frac{-\theta}{q-1}} \varepsilon^{\frac{-\alpha(q-\theta-1)}{q-1}} \int_{0}^{T} \int_{\Omega^{2 \varepsilon} \backslash \Omega^{\varepsilon}} d x d t+C \lambda^{\frac{\theta p}{r}} \varepsilon^{\frac{-\alpha(r-\theta)-\theta p}{r}} \int_{0}^{T} \int_{\Omega^{2 \varepsilon} \backslash \Omega^{\varepsilon}} d x d t \\
& +C \varepsilon^{\frac{\alpha[(p-s)(\theta-q)+s]-\beta \theta p}{q(p-s)-s}} \int_{0}^{T} \int_{\Omega^{2 \varepsilon} \backslash \Omega^{\varepsilon}} d x d t \\
\leq & C T^{\frac{q-\theta-1}{q-1}} \varepsilon^{\frac{\alpha \theta-(q-1)(\alpha-1)}{q-1}}+C T \varepsilon^{\frac{r(1-\alpha)+\theta(\alpha-p)}{r}}+C T \varepsilon^{\frac{\alpha[(p-s)(\theta-q)+s]-\beta \theta p+q(p-s)-s}{q(p-s)-s}} \\
= & C T^{\frac{q-\theta-1}{q-1}} \varepsilon^{\sigma_{1}}+C T \varepsilon^{\sigma_{2}}+C T \varepsilon^{\sigma_{3},}
\end{aligned}
$$

where

$$
\begin{aligned}
& \sigma_{1}=\frac{\alpha \theta-(q-1)(\alpha-1)}{q-1}, \quad \sigma_{2}=\frac{r(1-\alpha)+\theta(\alpha-p)}{r}, \\
& \sigma_{3}=\frac{\alpha[(p-s)(\theta-q)+s]-\beta \theta p+q(p-s)-s}{q(p-s)-s} .
\end{aligned}
$$

By the conditions in (I) of Theorem 1 , we have $\sigma_{1}>0, \sigma_{2}>0$, and $\sigma_{3}>0$. Then, letting $\varepsilon \rightarrow 0$ and combining with a random selection of $T$ yield

$$
\int_{\mathbf{S}} a(x) u^{\theta} d x d t=0
$$

and hence we get $u(x, t)=0$ a.e. in $\mathbf{S}$.

Step 3. When both $a(x)$ and $b(x)$ are singular at $\{0\}$, take $\Omega=\mathbf{R}^{N}$.

Choosing $\xi=\xi_{R}$ in (3.7), one has

$$
\operatorname{supp}|\nabla \xi|=B_{2 R}(0) \backslash B_{R}(0)=\{x \in \Omega: R \leq|x| \leq 2 R\} .
$$

It follows from (2.3) that

$$
\begin{aligned}
\int_{0}^{T} \int_{\mathbf{R}^{N}} a(x) u^{\theta} \chi^{k} d x d t \leq & C T^{\frac{-\theta}{q-1}} \int_{0}^{T} \int_{B_{2 R}(0) \backslash B_{R}(0)}|x|^{\frac{-\alpha(q-\theta-1)}{q-1}} d x d t \\
& +C \lambda^{\frac{\theta p}{r}} \int_{0}^{T} \int_{B_{2 R}(0) \backslash B_{R}(0)}|x|^{\frac{-\alpha(r-\theta)}{r}}\left|\nabla \xi_{R}\right|^{\frac{\theta p}{r}} d x d t \\
& +C \int_{0}^{T} \int_{B_{2 R}(0) \backslash B_{R}(0)}^{|x|^{\frac{\alpha[(p-s)(\theta-q)+s]-\beta \theta p}{q(p-s)-s}} .}
\end{aligned}
$$

Combining the above result with (2.5), we get

$$
\begin{aligned}
& \int_{0}^{T} \int_{B_{R}(0)} a(x) u^{\theta} d x d t \\
& \leq \int_{0}^{T} \int_{\mathbf{R}^{N}} a(x) u^{\theta} \chi^{k} d x d t \\
& \leq C T^{\frac{-\theta}{q-1}} \int_{0}^{T} \int_{B_{2 R}(0) \backslash B_{R}(0)}|x|^{\frac{-\alpha(q-\theta-1)}{q-1}} d x d t \\
& \quad+C \lambda^{\frac{\theta p}{r}} \int_{0}^{T} \int_{B_{2 R}(0) \backslash B_{R}(0)}|x|^{\frac{-\alpha(r-\theta)}{r}}\left|\nabla \xi_{R}\right|^{\frac{\theta p}{r}} d x d t
\end{aligned}
$$




$$
\begin{aligned}
& +C \int_{0}^{T} \int_{B_{2 R}(0) \backslash B_{R}(0)}^{|x|^{\frac{\alpha[(p-s)(\theta-q)+s]-\beta \theta p}{q(p-s)-s}}} \\
& =C T^{\frac{-\theta}{q-1}} \int_{0}^{T} \int_{\{x \in \Omega: R \leq|x| \leq 2 R\}}|x|^{\frac{-\alpha(q-\theta-1)}{q-1}} d x d t \\
& +C \lambda^{\frac{\theta p}{r}} \int_{0}^{T} \int_{\{x \in \Omega: R \leq|x| \leq 2 R\}}|x|^{\frac{-\alpha(r-\theta)}{r}}\left|\nabla \xi_{R}\right|^{\frac{\theta p}{r}} d x d t \\
& +C \int_{0}^{T} \int_{\{x \in \Omega: R \leq|x| \leq 2 R\}}|x|^{\frac{\alpha[(p-s)(\theta-q)+s]-\beta \theta p}{q(p-s)-s}} d x d t \\
& \leq C T^{\frac{-\theta}{q-1}} R^{\frac{-\alpha(q-\theta-1)}{q-1}} \int_{0}^{T} \int_{B_{2 R}(0) \backslash B_{R}(0)} d x d t+C R^{\frac{-\alpha(r-\theta)-\theta p}{r}} \int_{0}^{T} \int_{B_{2 R}(0) \backslash B_{R}(0)} d x d t \\
& +R^{\frac{\alpha[(p-s)(\theta-q)+s]-\beta \theta p}{q(p-s)-s}} \int_{0}^{T} \int_{B_{2 R}(0) \backslash B_{R}(0)} d x d t \\
& \leq C T^{\frac{q-\theta-1}{q-1}} R^{N-\frac{\alpha(q-\theta-1)}{q-1}}+C T R^{N-\frac{\alpha(r-\theta)+\theta p}{r}}+C T R^{N-\frac{\alpha[(p-s)(\theta-q)+s]+\beta \theta p}{q(p-s)-s}} \\
& =C T^{\frac{-\theta}{q-1}} R^{\sigma_{4}}+C T R^{\sigma_{5}}+C T R^{\sigma_{6}} \text {, }
\end{aligned}
$$

where

$$
\begin{aligned}
& \sigma_{4}=N-\frac{\alpha(q-\theta-1)}{q-1}, \quad \sigma_{5}=N-\frac{\alpha(r-\theta)+\theta p}{r}, \\
& \sigma_{6}=N+\frac{\alpha[(p-s)(\theta-q)+s]-\beta \theta p}{q(p-s)-s} .
\end{aligned}
$$

If one of the conditions (i) and (ii) in (II) of Theorem 1 holds, we have $\sigma_{4}<0, \sigma_{5}<0$, and $\sigma_{6}<0$. Then, letting $R \rightarrow \infty$ and combining with a random selection of $T$, we finally arrive at

$$
\int_{\mathbf{S}} a(x) u^{\theta} d x d t=0
$$

Therefore, we obtain $u(x, t)=0$ a.e. in $\mathbf{S}$, and the proof is completed.

\section{The proof of Theorem 2}

In this proof, we use the method of contradiction. We take the same test function as in Section 3 and the proof is similar to that of Theorem 1. Suppose there exists $u$ such that $u \geq c>0$.

(i) Assume that both $a(x)$ and $b(x)$ are singular on $\partial \Omega$. It follows from (2.2), (3.7), and (3.10) that

$$
\int_{0}^{T} \int_{\Omega^{2 \varepsilon} \backslash \Omega^{\varepsilon}} \rho^{-\alpha}(x) u^{\theta} \chi^{k} d x d t \leq C T^{\frac{q-\theta-1}{q-1}} \varepsilon^{\sigma_{1}}+C T \varepsilon^{\sigma_{2}}+C T \varepsilon^{\sigma_{3}} .
$$

By the definition of $\chi$ and the assumption $u \geq c>0$, we have

$$
C T^{\frac{q-\theta-1}{q-1}} \varepsilon^{\sigma_{1}}+C T \varepsilon^{\sigma_{2}}+C T \varepsilon^{\sigma_{3}} \geq C T \varepsilon^{1-\alpha} .
$$


Then

$$
\max \left\{T^{\frac{-\theta}{q-1}} \varepsilon^{\sigma_{1}}, \varepsilon^{\sigma_{2}}, \varepsilon^{\sigma_{3}}\right\} \geq \varepsilon^{1-\alpha}
$$

i.e., one of following inequalities holds:

$$
T^{\frac{-\theta}{q-1}} \geq \varepsilon^{1-\alpha-\sigma_{1}}, \quad \varepsilon^{\sigma_{2}} \geq \varepsilon^{1-\alpha}, \quad \varepsilon^{\sigma_{3}} \geq \varepsilon^{1-\alpha} .
$$

Since $0<\varepsilon<1$, if $\alpha>p$ or $\beta<\alpha-\frac{\alpha s}{p}$, and $T>1$ is large enough and $\varepsilon$ is sufficiently small, all inequalities in (4.1) do not hold, which is a contradiction.

(ii) Assume that both $a(x)$ and $b(x)$ are singular at 0. By combining (2.3) and (3.7) with (3.14), we get the inequality

$$
\int_{0}^{T} \int_{B_{R}(0)}|x|^{-\alpha} u^{\theta} d x d t \leq C T^{\frac{q-1-\theta}{q-1}} R^{\sigma_{4}}+C T R^{\sigma_{5}}+C T R^{\sigma_{6}} .
$$

Combining this inequality with $u \geq c>0$, we have

$$
C T^{\frac{-\theta}{q-1}} R^{\sigma_{4}}+C T R^{\sigma_{5}}+C T R^{\sigma_{6}} \geq C T R^{N-\alpha},
$$

i.e., $\max \left\{T^{\frac{-\theta}{q-1}} R^{\sigma_{4}}, R^{\sigma_{5}}, R^{\sigma_{6}}\right\} \geq R^{N-\alpha}$. Clearly, we have the inequalities

$$
R^{\sigma_{5}} \geq R^{N-\alpha}, \quad R^{\sigma_{6}} \geq R^{N-\alpha} .
$$

If $\alpha<p$ or $\beta>\alpha-\frac{\alpha s}{p}$, the inequalities in (4.2) do not hold, which is a contradiction. The proof is completed.

\section{Competing interests}

The authors declare that they have no competing interests.

\section{Authors' contributions}

All authors contributed equally to the manuscript and read and approved the final manuscript.

\section{Acknowledgements}

This work is supported by the Natural Science Foundation of Shandong Province of China (ZR2012AM018) and the Fundamental Research Funds for the Central Universities (No. 201362032). The authors would like to sincerely thank all the reviewers for their insightful and constructive comments.

Received: 31 October 2013 Accepted: 30 January 2014 Published: 11 Feb 2014

\section{References}

1. Bebernes, J, Eberly, D: Mathematical Problems from Combustion Theory. Springer, New York (1989)

2. Samarskii, AA, Galaktionov, VA, Kurdyumov, SP, Mikhailov, AP: Blow-up in Quasilinear Parabolic Equations. de Gruyter, Berlin (1995)

3. Wu, Z, Zhao, J, Yin, J: Nonlinear Diffusion Equations. World Scientific, Singapore (2001)

4. Fujita, H: On the blowing up of solutions to the Cauchy problem for $u_{t}=\Delta u+u^{1+\alpha}$. J. Fac. Sci., Univ. Tokyo, Sect. $1 \mathrm{~A}$, Math. 13, 119-124 (1966)

5. Galaktionov, VA, Vazquez, JL: Continuation of blow up solutions of nonlinear heat equations in several space dimensions. Commun. Pure Appl. Math. 50(1), 1-67 (1997)

6. Kartsatos, AG, Kurta, $\mathrm{V}$ : On the critical Fujita exponents for solutions of first-order nonlinear evolution inequalities. J. Math. Anal. Appl. 269, 73-86 (2002)

7. Piccirillo, AM, Toscano, L, Toscano, S: Blow-up results for a class of first-order nonlinear evolution inequalities. Differ. Equ. 212, 319-350 (2005)

8. Jiang, ZX, Zheng, SM: A Liouville-type theorem for a doubly degenerate parabolic inequality. J. Math. Phys. 30A(3), 639-643 (2010)

9. Mitidieri, E, Pohozaev, SI: Nonexistence of positive solution for quasilinear elliptic problems on $\mathbf{R}^{N}$. Proc. Steklov Inst. Math. 227, 192-222 (1999) 
10. Mitidieri, E, Pohozaev, SI: Nonexistence of weak solutions for some degenerate elliptic and parabolic problems on $\mathbf{R}^{N}$. J. Evol. Equ. 1, 159-220 (2001)

11. Wei, GM: Nonexistence of global solutions for evolutional $p$-Laplace inequalities with singular coefficients. J. Math. Anal. Appl. 28A(2), 387-394 (2007)

12. Mitidieri, E, Pohozaev, SI: A priori estimates and the absence of solutions of nonlinear partial differential equations and inequalities. Proc. Steklov Inst. Math. 234, 1-362 (2001)

13. Karisti, G: On the absence of solutions of systems of quasilinear elliptic inequalities. Differ. Equ. 38, 375-383 (2002)

14. Filippucci, R: Nonexistence of positive weak solutions of elliptic inequalities. Nonlinear Anal. 70(8), 2903-2916 (2009)

15. Galakhov, El: Some nonexistence results for quasi-linear PDE's. Commun. Pure Appl. Anal. 6, 141-161 (2007)

16. Li, XH, Li, FQ: A priori estimates for nonlinear differential inequalities and applications. J. Math. Anal. Appl. 378, 723-733 (2011)

17. Li, XH, Li, FQ: Nonexistence of solutions for singular quasilinear differential inequalities with a gradient nonlinearity. Nonlinear Anal. 75, 2812-2822 (2012)

18. Bandle, C, Essen, M: On positive solutions of Emden equations in cone-like domains. Arch. Ration. Mech. Anal. 4 319-338 (1990)

19. Laptev, GG: Absence of solutions to semilinear parabolic differential inequalities in cones. Mat. Sb. 192, 51-70 (2001)

20. Fang, ZB, Chao, F, Zhang, LJ: Liouville theorems of slow diffusion differential inequalities with variable coefficients in cone. J. Korean Soc. Ind. Appl. Math. 15(1), 43-55 (2011)

$10.1186 / 1029-242 X-2014-62$

Cite this article as: Fang and Xu: Liouville theorems for a singular parabolic differential inequality with a gradient term. Journal of Inequalities and Applications 2014, 2014:62

\section{Submit your manuscript to a SpringerOpen ${ }^{\circ}$ journal and benefit from:}

- Convenient online submission

Rigorous peer review

- Immediate publication on acceptance

- Open access: articles freely available online

- High visibility within the field

- Retaining the copyright to your article 